\title{
Nasolabial Cyst in an Elderly Patient: Report of a Case and Literature Review
}

\author{
Takaki Iwagami',2, Yoshihiro Morita1,2,3*, Atsuko Niki-Yonekawa',2, Yukiko Kusuyama1,2, \\ Nobuo Morita ${ }^{1,4}$
}

\author{
${ }^{1}$ Department of Oral and Maxillofacial Surgery, Seichokai Hannan Municipal Hospital, Hannan City, Japan \\ ${ }^{2}$ Departments of Oral and Maxillofacial Surgery II, Osaka University Graduate School of Dentistry, Osaka City, Japan \\ ${ }^{3}$ Stephenson Cancer Center, University of Oklahoma Health Science Center, Oklahoma, USA \\ ${ }^{4}$ Department of Oral-Maxillofacial Surgery, NS Medical \& Healthcare Service General Incorporation Foundation, \\ Wakayama City, Japan \\ Email: *yoshihiro-morita@ouhsc.edu
}

How to cite this paper: Iwagami, T., Morita, Y., Niki-Yonekawa, A., Kusuyama, Y. and Morita, N. (2017) Nasolabial Cyst in an Elderly Patient: Report of a Case and Literature Review. Open Journal of Stomatology, 7, 455-461.

https://doi.org/10.4236/ojst.2017.710040

Received: September 22, 2017

Accepted: October 20, 2017

Published: October 23, 2017

Copyright $\odot 2017$ by authors and Scientific Research Publishing Inc. This work is licensed under the Creative Commons Attribution International License (CC BY 4.0).

http://creativecommons.org/licenses/by/4.0/

\begin{abstract}
Nasolabial cysts are uncommonly diagnosed non-odontogenic soft tissue lesions occurring close to the nasal alar region of the face. Patients usually present with a slowly enlarging asymptomatic swelling. Diagnosis is usually made in the early stages because of the esthetic effects. Histologically, the lesion is lined with non-keratinized squamous epithelium or, more frequently, pseudostratified columnar epithelium with goblet cells. These cysts are most often diagnosed in the fourth decade of life. However, we report a case of nasolabial cyst in an 80-year-old woman, and discuss the diagnosis, differential diagnosis, and treatment with reference to the literature.
\end{abstract}

\section{Keywords}

Nasolabial Cyst, Klestadt Cyst, Pseudostratified Columnar Epithelium, Goblet Cell

\section{Introduction}

Nasolabial cyst is a rare non-odontogenic cyst originating in the maxillofacial soft tissues close to the nasal alar region of the face. The patient usually presents with a slowly enlarging asymptomatic swelling [1] [2] [3] [4]. The cyst is usually unilateral, and is often confused with other fissural or odontogenic cysts [4] [5]. Previous reports have suggested that this entity shows higher incidences on the left side and among women [6]. Diagnosis is usually made in the early stage, because of the obvious esthetic effects. Otherwise, nasolabial cyst is likely to remain 
undetected. This pathology may therefore be more frequent than previously thought [4] [6].

Swelling of the upper lip adjacent to the ala of the nose and a very typical facial asymmetry are the characteristic symptoms. Pain can occur if the cyst becomes infected [5] [7].

These cysts are usually diagnosed in the fourth or fifth decade of life [4]. However, we describe a case involving an 80-year-old woman who reported to our department with a chief complaint of swelling lateral to the right ala of the nose.

\section{Case Report}

An 80-year-old woman was referred to our outpatient clinic for evaluation and treatment of a unilateral swelling of unknown etiology in the right nasolabial region. She had noticed a slight swelling in that area several years earlier, and had since noticed a gradual increase in the size and painfulness of the swelling. Her past medical and family histories were unremarkable.

Physical examination revealed a painful, soft, round swelling under the right alar base. The swollen area was palpated intraorally, and was elastic and hard. The floor of the nose was swollen and narrowed from the affected side by the mass. The overlying skin was smooth, and overlying mucosa appeared normal. The lesion was an ovoid swelling measuring $32 \mathrm{~cm}$ (Figure 1). Computed tomography (CT) revealed a non-odontogenic cyst in the nasolabial area (Figure 2). Findings from blood and serum biochemistry were within normal limits.

The lesion was removed via an intraoral incision on the upper alveolar crest under general anesthesia. After raising a mucoperiosteal flap, the lesion was easily removed from the bone. Tight adhesions with the mucoperiosteal and overlying nasal mucosa were seen, but the mass was removed intact. Once the lesion was removed, the mucosa of the nasal floor was perforated and the defect was therefore closed with absorbable sutures.

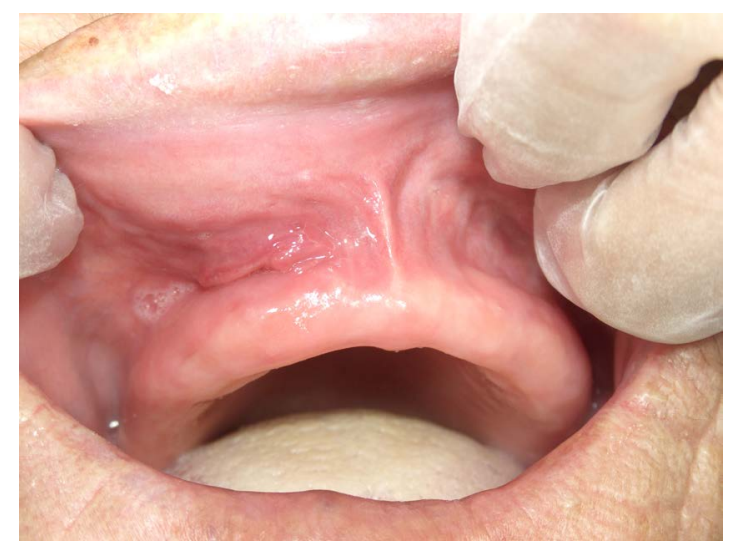

Figure 1. Preoperative intraoral view of the nasolabial cyst. The anterior floor of the mouth is distended by the submucosal cystic mass, which is covered by normal mucosa. 


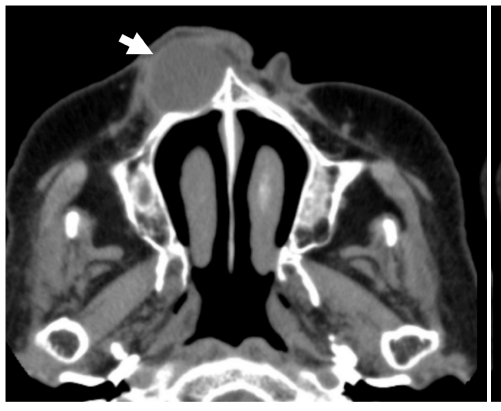

(a)

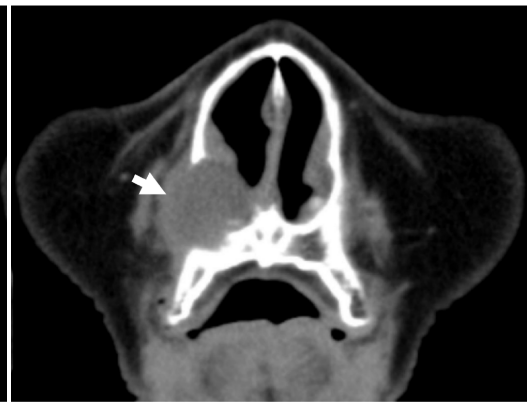

(b)

Figure 2. Axial (a) and coronal (b) CT reveals a well-demarcated, low-density cystic lesion (arrow).

Histopathologic examination revealed pseudostratified columnar epithelium with scattered goblet cells lining the cyst walls (Figure 3). The lesion was diagnosed as nasolabial cyst.

Symptoms resolved postoperatively, and follow-up after 10 months showed good healing without evidence of recurrence. The patient has given her consent for this case report to be published.

\section{Discussion}

Nasolabial cyst was first described by Zuchercandl in 1892 [8]. These nonodontogenic masses can be seen in the maxillofacial area. In the literature, the lesions have also been termed Klestadt cyst, nasoalveolar cyst, nasal vestibule cyst, nasal wing cyst, and mucoid cyst of the nose [8] [9]. Nasolabial cyst has been defined as a lesion located entirely within soft tissue, differing from nasoalveolar cyst, which causes maxillary bone erosion [3].

The lesion is submucosal and extraosseous, and expands via the gingivobuccal sulcus, pushing all the surround soft tissues outwards. These lesions are uncommon developmental entities that account for $0.7 \%$ of all maxillofacial cysts [7] [10].

Diagnosis is usually made in the fourth decade of life, with a noticeable predilection for women (female-to-male ratio, 3:1) [2] [5] [6] [10] [11]. A search of recent case reports revealed cases involving patients of 32-73 years old (Table 1). Most of the cases were female and there was no recurrence after surgery in the all cases. However, our case involved an 80-year-old woman.

The pathogenesis of nasolabial cyst remains uncertain. The lesion seems to be developmental, rather than inflammatory, in origin [11]. However, several theories on the pathogenesis of nasolabial cyst have been advanced, and the etiology is still debated [4]. The two more-popular hypotheses are that the cyst is either a fissural cyst arising from the epithelial rests along fusion lines of the globular, lateral, nasal, and maxillary processes or originates from remnants of the embryonic nasolacrimal ducts [3] [4]. Given the location of the cyst in the present case, the first theory seems more acceptable. 


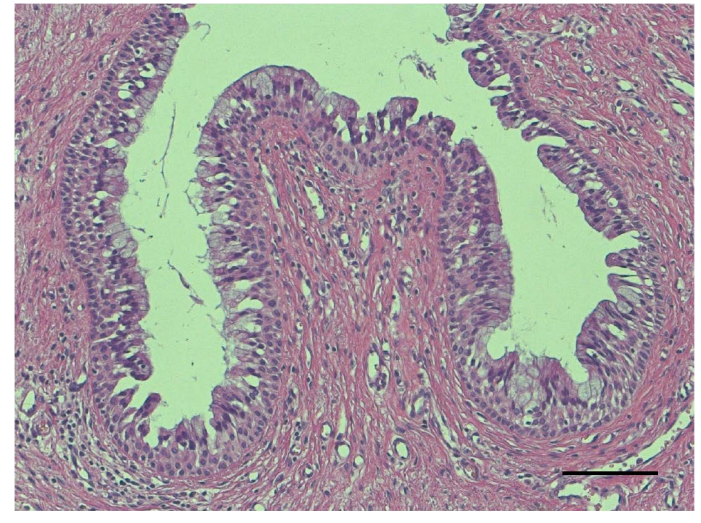

Figure 3. Epithelial lining of the cyst comprises pseudostratified columnar cells with scattered goblet cells (hematoxylin and eosin stain; scale bar, $100 \mu \mathrm{m}$; original magnification $200 \times$ ).

Table 1. The lis of reported cases of Nasolabial cyst beween 1997-2017.

\begin{tabular}{lclc}
\hline \multicolumn{1}{c}{ Reference } & Age & Gender & Outcome \\
\hline F López-Ríos et al., 1997 [1] & 44 & Female & Unknown \\
Nixdorf et al., 2003 [11] & 46 & Female & Good \\
Kyrmizakis et al., 2005 [15] & 48 & Female & Good \\
Iida et al., 2006 [2] & 47,54 & Female & Good \\
Patil et al., 2007 [16] & 30 & Female & Good \\
Tiago et al., 2008 [3] & $20-67$ & Female & Good \\
Aquilino et al., 2008 [17] & 48 & Female & Good \\
Marcoviceanu et al., 2009 [7] & 48 & Female & Light hypaesthesia (right upper lip) \\
Sahin, 2009 [13] & 53 & Male & Good \\
Kamal et al., 2011 [12] & 36 & Male & Unknown \\
Rallan et al., 2013 [18] & 32 & Female & Good \\
Parwani et al., 2013 [19] & 69 & Female & Unknown \\
Paramjit et al., 2014 [20] & 35 & Female & Good \\
Vinayak et al., 2015 [21] & 73 & Female & Unknown \\
Sato et al., 2016 [22] & 67 & Female & Good \\
Ocak et al., 2017 [23] & 54 & Female & Unknown \\
Our case, 2017 & $\mathbf{8 0}$ & Female & Good \\
\hline
\end{tabular}

However, our case showed the typical clinical features of a nasolabial cyst. The lesion is slow-growing, presenting as a fluctuant, painless, soft tissue swelling between the upper lip and nasal aperture. The swelling produces facial asymmetry with elevation of the nasal ala and inferior turbinate and obliteration of the nasolabial fold [2] [5] [6] [7] [10] [11]. Many nasolabial cysts probably remain undetected for several years because of the slow growth, until they become infected and painful or become associated with marked facial asymmetry [2]. 
Sometimes, the cyst may be identified incidentally on routine examination. When infected, the cyst enlarges rapidly, becomes tender, and may be mistaken for abscess of the nasal floor [6]. Extraorally, patients usually complain of swelling adjacent to the nose. Intraorally, the swelling usually causes discomfort with the use of dentures [12]. In our case, the swelling gradually increased along with associated inflammatory pain.

The differential diagnoses to be considered include central line cyst, cyst of the maxilla, odontogenic cyst, periapical cyst, periapical abscess, periapical granuloma, epidermal inclusion cyst, furunculosis of the base of the nose, and neoplasms of base of the nose [13]. For differentiation from these other lesions, the safety of the teeth in the nasolabial region is clinically important. Radiological examination is crucial in differentiating odontogenic and non-odontogenic cysts in this region. Bone erosion is not expected, particularly in the early stages of the disease [13]. Clinical, radiological and histopathological examinations can achieve diagnosis of this lesion.

CT reveals a well-demarcated, low-density cystic lesion in the anterolateral side of the piriform aperture. There are usually no signs of bone invasion, but remodeling of the underlying anterior maxilla may occur [3] [4] [6] [10]. Magnetic resonance imaging demonstrates nasolabial cyst with homogeneous intermediate-intensity T1 signals and homogeneous high-intensity T2 signals [3] [6].

Definitive diagnosis of the lesion is obtained by correlating clinical, radiological, and histopathological examinations. The histological features consist of a cyst lined by epithelium of varying characteristics, ranging from mundane stratified non-keratinized squamous epithelium to pseudostratified columnar epithelium. Cilia and scattered mucous or goblet cells may be present. Apocrine changes have also been reported in the cyst lining [14]. Most often, the cyst wall comprises fibrous connective tissue with an occasional skeletal muscle bundle. Most often, the contents of the cyst include thin mucoid or straw-colored serous fluid. However, purulent material dominates when the cyst is infected secondarily [14].

The classic and preferred treatment of nasolabial cysts is surgical excision via a sublabial incision. The cyst wall should be carefully dissected from the surrounding tissues and removed, paying attention to tight attachments between the cyst and nasal mucosa. [4] If perforation of the floor of the nasal cavity arises, repair of the defect with primarily absorbable suture is necessitated, as in our case [4].

\section{Conclusion}

Nasolabial cyst is an uncommon, benign cystic lesion appearing as a soft tissue mass near the nasal alae. Nasolabial cysts can probably remain undetected for several years until symptoms appear. Surgical enucleation is the treatment of choice and is associated with a low recurrence rate. Nasolabial cyst should be considered among the differential diagnoses of soft tissue vestibular swelling in the nasal vestibule, nasal base, and sublabial area. Diagnosis for nasolabial cyst is 
usually made in 40 - 50 years old, thus detection in an elderly patient of 80 or more years old is uncommon. However, for precise diagnosis and surgery, it is very important to realize that it cannot be improbable that it will be developed or discovered after age 80 as in our reported case.

\section{References}

[1] López-Ríos, F., Lassaletta-Atienza, L., Domingo-Carrasco, C. and Martinez-Tello, F.J. (1997) Nasolabial Cyst: Report of a Case with Extensive Apocrine Change. Oral Surgery, Oral Medicine, Oral Pathology, Oral Radiology, and Endodontology, 84, 404-406. https://doi.org/10.1016/S1079-2104(97)90039-1

[2] Iida, S., Aikawa, T., Kishino, M., et al. (2006) Spheric Mass Beneath the Alar Base: MR Images of Nasolabial Cyst and Schwannoma. American Journal of Neuroradiology, 27, 1826-1829.

[3] Tiago, R.S.L., Maia, M.S., do Nascimento, G.M.S., Correa, J.P. and Salgado, D.C. (2008) Nasolabial Cyst: Diagnostic and Therapeutical Aspects. Brazilian Journal of Otorhinolaryngology, 74, 39-43. https://doi.org/10.1016/S1808-8694(15)30749-7

[4] Boffano, P., Gallesio, C., Campisi, P. and Roccia, F. (2011) Diagnosis and Surgical Treatment of a Nasolabial Cyst. Journal of Craniofacial Surgery, 22, 1946-1948. https://doi.org/10.1097/SCS.0b013e31822ea751

[5] Su, C.-Y., Huang, H.-T., Liu, H.-Y., Huang, C.-C. and Chien, C.-Y. (2006) Scanning Electron Microscopic Study of the Nasolabial Cyst: Its Clinical and Embryological Implications. Laryngoscope, 116, 307-311. https://doi.org/10.1097/01.mlg.0000199598.37461.8e

[6] Yuen, H.-W., Julian, C.-Y.L. and Samuel, C.-L.Y. (2007) Nasolabial Cysts: Clinical Features, Diagnosis, and Treatment. British Journal of Oral and Maxillofacial Surgery, 45, 293-297. https://doi.org/10.1016/j.bjoms.2006.08.012

[7] Marcoviceanu, M.P., Metzger, M.C., Deppe, H., et al. (2009) Report of Rare Bilateral Nasolabial Cysts. Journal of Cranio-Maxillo-Facial Surgery, 37, 83-86. https://doi.org/10.1016/j.jcms.2008.11.006

[8] Kuriloff, D.B. (1987) The Nasolabial Cyst-Nasal Hamartoma. Otolaryngology-Head and Neck Surgery, 96, 268-272. https://doi.org/10.1177/019459988709600307

[9] Cohen, M.A. and Hertzanu, Y. (1985) Huge Growth Potential of the Nasolabial Cyst. Oral Surgery, Oral Medicine, Oral Pathology, Oral Radiology, 59, 441-445. https://doi.org/10.1016/0030-4220(85)90077-5

[10] Amaral, T.M.P., de Freitas, J.B., de Fátima da Conceição, J., de Aguiar, M.C.F., da Silva Fonseca, L.M. and Mesquita, R.A. (2005) Nasolabial Cyst with Radiographic Contrast Medium: Report of Two Cases. Dentomaxillofacial Radiology, 34, 256-258. https://doi.org/10.1259/dmfr/30955495

[11] Nixdorf, D.R., Peters, E. and Lung, K.E. (2003) Clinical Presentation and Differential Diagnosis of Nasolabial Cyst. Journal of the Canadian Dental Association, 69, 146-149.

[12] Kamal, R., Dahiya, P. and Palaskar, S. (2011) Klestadt's Cyst. Journal of Natural Science, Biology and Medicine, 2, 128-130. https://doi.org/10.4103/0976-9668.82304

[13] Sahin, C. (2009) Nasolabial Cyst. Case Reports in Medicine, 2009, Article ID: 586201. https://doi.org/10.1155/2009/586201

[14] Perez, A.J. and Castle, J.T. (2013) Nasolabial Cyst. Head and Neck Pathology, 7, 155-158. https://doi.org/10.1007/s12105-013-0424-5 
[15] Kyrmizakis, D.E., Lachanas, V.A., Benakis, A.A., Velegrakis, G.A. and Aslanides, I.M. (2005) Bilateral Nasolabial Cysts Associated with Recurrent Dacryocystitis. The Journal of Laryngology \& Otology, 119, 412-414. https://doi.org/10.1258/0022215053945787

[16] Patil, K., Mahima, V.G. and Divya, A. (2007) Klestadt's Cyst: A Rarity. Indian Journal of Dental Research, 18, 23-26. https://doi.org/10.4103/0970-9290.30918

[17] Aquilino, R.N., Bazzo, V.J., Faria, R.J.A., Eid, N.L.M. and Bóscolo, F.N. (2008) Nasolabial Cyst: Presentation of a Clinical Case with CT and MR Images. Brazilian Journal of Otorhinolaryngology, 74, 467-471.

[18] Rallan, N.S., Rallan, M., Singh, N.N. and Gadiputi, S. (2013) Nasolabial Cyst Mimicking Inflammatory Cyst. BMJ Case Reports, 2013.

[19] Parwani, R., Parwani, S. and Wanjari, S. (2013) Diagnosis and Management of Bilateral Nasolabial Cysts. Journal of Oral and Maxillofacial Pathology, 17, 443-446. https://doi.org/10.4103/0973-029X.125217

[20] Paramjit, K., Jeevan, L. and Reecha, A. (2014) Nasolabial Cyst: Review of Literature and a Case Report. Journal of Maxillofacial and Oral Surgery, 13, 227-230. https://doi.org/10.1007/s12663-010-0108-6

[21] Vinayak, K.M. and Ruchi, M. (2015) A Rare Case of Nasolabial Cyst; A Case Report. International Journal of Applied Dental Sciences, 1, 13-15.

[22] Masaru, S., Keiichi, M., Yuji, K. and Hiroyuki, H. (2016) Bilateral Nasolabial Cysts: A Case Report. Journal of Medical Case Reports, 10, 246. https://doi.org/10.1186/s13256-016-1024-2

[23] Ali, O., Suayip, B.D., Ibrahim, S.B. and Binali, C. (2017) Nasolabial Cyst: A Case Report with Ultrasonography and Magnetic Resonance Imaging Findings. Case Reports in Dentistry, 2017, Article ID: 4687409. 